\title{
Clinical, Haemato-Biochemical and Molecular Findings of Babesiosis in Dogs
}

\author{
Juripriya Brahma ${ }^{*}$, D. Chandrasekaran ${ }^{2}$, M.G. Jayathangaraj ${ }^{1}$, \\ S. Vairamuthu ${ }^{3}$ and C. Soundararajan ${ }^{4}$ \\ ${ }^{1}$ Department of Veterinary Clinical Medicine, ${ }^{2}$ Department of Clinics, ${ }^{3}$ Department of \\ Centralised Clinical Laboratory, ${ }^{4}$ Department of Veterinary Parasitology, Madras Veterinary \\ College, TANUVAS, Chennai-600007, India \\ *Corresponding author
}

\section{A B S T R A C T}

\section{Keywords}

Babesia species, Multiplex PCR, Dog, Giemsa staining

Article Info

Accepted:

14 December 2018 Available Online: 10 January 2019
Canine babesiosis is a hemoprotozoan parasite affecting dogs. The goal of this study was to provide an overview of molecular examination of babesiosis and heamato-biochemical changes in canine babesiosis infected dogs. In this study, 8 cases infected with Babesia were confirmed by means of hematological, biochemical and multiplex PCR. The most common clinical signs were anorexia, pale or icteric mucous membranes, high rise of temperature and dark urine colour. The haematological and biochemical parameters showed decrease level of RBC, Hb, PCV, Platelets level and increase level of WBC, ALT, ALP, Total bilirubin, BUN and creatinine value.

\section{Introduction}

Babesiosis is a life-threatening disease of dogs that is caused by hemoprotozoan apicomplexan parasites of the genus Babesia. The disease is mainly caused by Babesia gibsoni (smaller piroplasms) and Babesia canis (larger piroplasms) and is transmitted by brown dog tick Rhipicephalus sanguineus. Dermacentor reticularus, Dermacentor marginatus and Haemaphysalis leachi also involved in the transmission of babesiosis (Filipe and Luciana, 2006).
Dogs become infected when ticks feed for 2-3 days and release sporozoites into the circulation (Taboada and Merchant, 1991, Taboada, 1998). Inside the host the organisms attach to the red cell membrane and are engulfed by endocytosis. In the cytoplasm, binary fission occurs, resulting in merozoites. Ticks become infected with merozoites during feeding and may remain infective for many generations through trans-stadial and transovarial transmission. Parasitaemia peaked at $1.9 \%$ to $6 \%$ by $4-6$ weeks after infection. Easily detectable parasitaemia was present for 3 to 4 weeks. The severity of 
clinical signs was highly variable and developed approximately 1 to 2 weeks after infection (Meinkoth et al., 2002).

After parasitaemia, the immune system does not totally eradicate the infection and chronic carrier state remains. Relapses may occur months to years later and long-term sequelae, such as glomerulonephritis or polyarthritis may develop (Conrad et al., 1991, Wozniak et al., 1997 and Lobetti 1998) with Babesia gibsoni infection, parasitemia is usually mild although anemia can be severe. Splenectomized animals may have more severe parasitemia and anemia.

Babesia gibsoni can cause hyperacute, acute and chronic infections. Acute infections are rare and primarily occur in puppies resulting in rapid death. These infections are presumed to be maternally acquired. Acute B. gibsoni infections are typically associated with fever, lethargy, thrombocytopenia and anemia. Chronic infections may be completely asymptomatic or may be characterized by intermittent fever, lethargy and weight loss.

Hemolytic anemia is the predominant feature of babesiosis and thrombocytopenia is also common in infected dogs. Anemia is attributed to extra and intravascular hemolysis. Mechanisms of RBC destruction include increased osmotic fragility, shortened RBC life span and erythrophagocytosis. Secondary immune-mediated destruction occurs because of parasite antigens on the RBC surface, parasite-induced membrane damage and possibly other membraneassociated antigens (Taboada and Merchant, 1991, Jacobson and Clark, 1994, Wozniak et al., 1997, Taboada, 1998). Oxidative damage, impaired hemoglobin function, sludging and sequestration of erythrocytes also occur (Taboada and Merchant, 1991, Jacobson and Clark, 1994, Taboada, 1998).
In addition to tickborne transmission, vertical transmission is also suspected and infections have been identified in a dam and her 3-day old puppies. Transmission can also occur through transmission of infected blood.

Diagnosis $B$. gibsoni infection can be challenging because many animals are presumed to have idiopathic immunemediated anemia or another tick borne disease. Detecting RBC auto-agglutination and positive results of a Coombs' test may complicate the diagnosis. Identifying the parasite through blood smear evaluation can be difficult because of the small size of the organism relatively low levels of parasitemia.

B. gibsoni is approximately $1 \times 2.5 \mu \mathrm{m}$ in size and signet, rod or cocci shape. Giemsa or Wright's stained of fresh blood smears are recommended. The organisms are found in the peripheral portion of the blood smear. The serological assays IFA (Immunofluorescent antibody) and ELISA (Enzyme-linked immunosorbent assay) are also been used to detect infection.

PCR (Polymerase chain reaction) is used for identifying the infective species, detecting low levels of parasitaemia, recognizing subclinical infections and monitoring response to therapy.

\section{Materials and Methods}

Thirty four dogs of different breeds, ages naturally infected with canine Babesia were selected from the Madras Veterinary College Teaching Hospital under this study. Blood samples was collected from animals exhibiting clinical symptoms of fever, pale mucous membrane, loss of appetite, depression, haemoglobinuria and tick infestation. Blood was collected in EDTAanticoagulated for complete blood counts, smear observations and PCR analysis. 
Complete blood count (CBC) was assessed with an automatic cell counter (Mindray BC Vet 2800). Parameters assessed were: red blood cell count (RBC), hemoglobin $(\mathrm{Hb})$, PCV, PLT count, white blood cell count (WBC), WBC differential count including neutrophils, lymphocytes, monocytes, eosinophil.

Blood smears were prepared, air dried and stained with Giemsa solution. Smears were examined under oil immersion objective (100X) microscope to detect the piroplasms and the results obtained were compared with PCR assay.

Regarding estimation of biochemical parameters $2 \mathrm{ml}$ of blood was collected without anticoagulant vial. The serum concentration of alanine amino transferase (ALT), alkaline phosphatase (ALP), blood urea nitrogen (BUN), blood glucose, albumin, creatinine, total bilirubin and direct bilirubin were determined by automated serum biochemistry analyser (A-15 Biosystem) by using standard kits. Results obtained were expressed as means \pm standard error.

\section{DNA extraction}

DNA isolation kits (QIAamp DNA Mini Kit ${ }^{\circ}$, Qiagen) was used for the extraction of parasite DNA from $200 \mu$ l of blood sample collected in EDTA vacutainers according to the manufacturer's instructions. Genomic DNA isolated from the whole blood of healthy dogs was used as negative control.

\section{Multiplex PCR amplification}

Multiplex PCR for amplification of the 16s rRNA gene fragment of genus Babesia and VirB9 of E. canis was employed by using the procedure of Kledmanee et al., (2009). The following primers were used (Table 1).

Thermocycling consisted initial denaturation step of 15 minutes at $94^{\circ} \mathrm{C}$ followed by 30 cycles of 45 second at $94^{\circ} \mathrm{C}, 45$ second at $65^{\circ} \mathrm{C}$ and 90 second at $72^{\circ} \mathrm{C}$ with final extension step of $10 \mathrm{~min}$ at $72^{\circ} \mathrm{C}$. The amplicons were separated by electrophoresis using $1.5 \%$ agarose gel in $40 \mathrm{mM}$ Tris-acetic acetate ( $\mathrm{pH}$ 8.4), $1 \mathrm{mM}$ EDTA stained with ethidium bromide $(0.5 \mu \mathrm{g} / \mathrm{ml})$ after that visualized under UV light.

\section{Results and Discussion}

A total of 8 dogs infected with canine babesiosis were diagnosed by clinical examination and observation of intra erythrocytic piroplasm within blood smears. Giemsa-stained blood smears shows the presence of small pear-shaped (Plate 1) parasites.

Animal infested with tick was observed (Plate $2)$. The most prevalent clinical abnormalities were anorexia, Pale or icteric mucous membranes (Plate 3a, 3b), lethargy (Plate 4), fever, dark urine and ecchymosis on ventral aspect of abdomen (Plate 5).

Table.1 PCR primers

\begin{tabular}{|l|l|l|l|}
\hline Pathogen & Primer & Sequence (5'-3') & $\begin{array}{l}\text { Product } \\
\text { Size }\end{array}$ \\
\hline Babesia & Ba103F & CCAATCCTGACACAGGGAGGTAGTGACA & \\
. & Ba721R & CCCCAGAACCCAAAGACTTTGATTTCTCTCAAG & $619 \mathrm{bp}$ \\
\hline \multirow{2}{*}{ E. canis } & Ehr1401F & CCATAAGCATAGCTGATAACCCTGTTACAA & \\
\cline { 2 - 3 } & Ehr1780R & TGGATAATAAAACCGTACTATGTATGCTAG & $380 \mathrm{bp}$ \\
\hline
\end{tabular}


Table. 2 Hematological parameters and biochemical parameters in 8 dogs infected with Canine babesiosis

\begin{tabular}{|l|l|}
\hline Parameters & Mean \pm SE \\
\hline Hb $(\mathbf{g} / \mathbf{d l})$ & $4.29 \pm 1.63$ \\
\hline PCV $(\mathbf{\%})$ & $13.03 \pm 0.58$ \\
\hline RBC $\left(\times \mathbf{1 0}^{\mathbf{6}} / \mathbf{m m}^{\mathbf{3}}\right)$ & $2.12 \pm 0.08$ \\
\hline WBC $\left(\mathbf{1 0}^{\mathbf{3}} / \mathbf{m m}^{\mathbf{3}}\right)$ & $8514.23 \pm 580.13$ \\
\hline Platelets $\left(\mathbf{1 0} / \mathbf{m m}^{\mathbf{3}}\right)$ & $139750 \pm 20490.39$ \\
\hline Neutrophil \% & $73.88 \pm 0.44$ \\
\hline Lymphocyte \% & $17.5 \pm 0.54$ \\
\hline Monocyte \% & $6.25 \pm 0.36$ \\
\hline Eosinophil \% & $2 \pm 0.07$ \\
\hline BUN (mg/dl) & $69.88 \pm 12.16$ \\
\hline Creatinine (mg/dl) & $1.6 \pm 0.30$ \\
\hline Calcium (mg/dl) & $10.23 \pm 0.22$ \\
\hline Phosphorus (mg/d) & $5.05 \pm 0.09$ \\
\hline ALT (U/L) & $113.76 \pm 11.44$ \\
\hline ALP (U/L) & $280.25 \pm 33.89$ \\
\hline Total bilirubin (mg/dl) & $0.96 \pm 0.12$ \\
\hline Direct bilirubin (mg/dl) & $0.49 \pm 0.036$ \\
\hline Total protein (g/d) & $5.93 \pm 0.22$ \\
\hline Albumin (g/dl) & $1.71 \pm 0.07$ \\
\hline Glucose (mg/dl) & $88.88 \pm 1.97$ \\
\hline
\end{tabular}
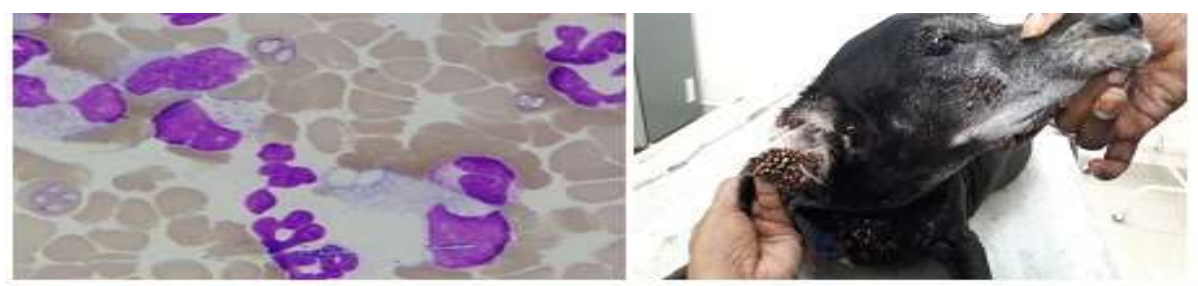

Platel : P icture showing intra erythrocytic Plate 2: Tick infestation pear-shaped form of Babesia canis.

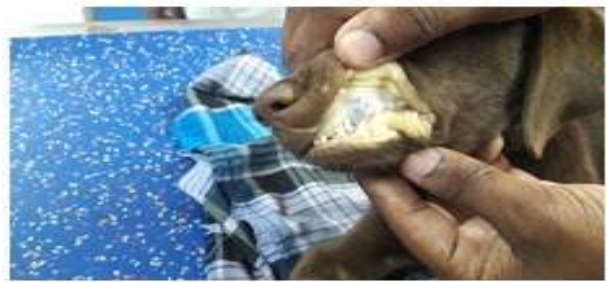

Plate 3a: A dog showing pale and icteric oral mucous membrane

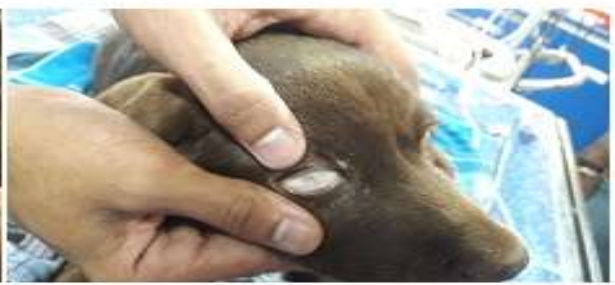

Plate 3b: A dog showing pale conjunctival mucous membrane

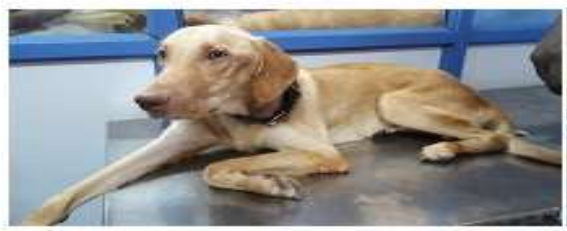

Plate 4: A dog showing weak and lethargy

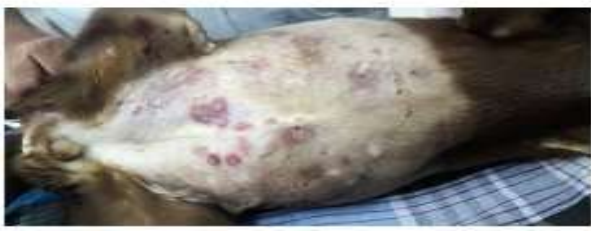

Plate 5: Ecchymosis on ventral aspect of $\angle I J U$ abdomen 


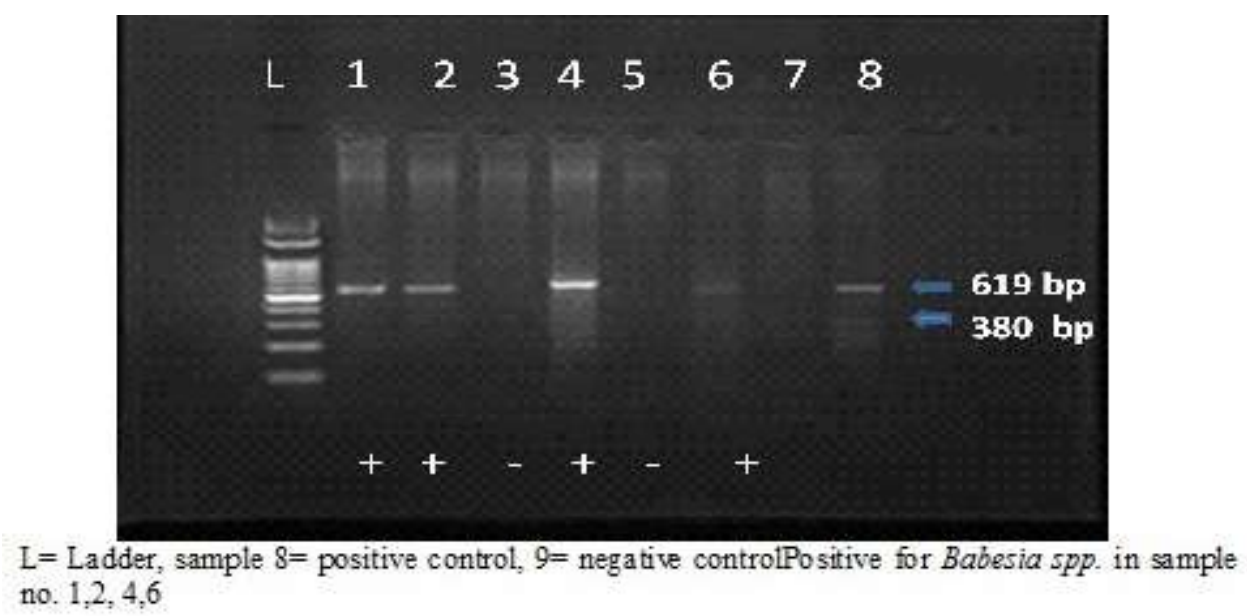

Plate 6: Babesia spp. positive in multiplex PCR

The finding revels that 8 dogs had the $\mathrm{RBC}, \mathrm{Hb}$, PCV and platelets values below the reference values and increase level of WBC value. In biochemical examination, ALT, ALP, Total bilirubin, BUN, Creatinine values are increase than the reference values (Table 2). As shown in (Plate.6) the primer Ba103F and Ba721R successfully amplified an approximately $619 \mathrm{bp}$ DNA fragment from all 8 dogs infected with canine babesiosis.

The goal of this study was to provide an overview of molecular examination of babesiosis and haematobiochemical changes.

In the present study, 8 cases infected with Babesia were confirmed by mean of clinical history, physical examination, hematological, biochemical and multiplex PCR. Anemia and thrombocytopenia were the most common hematological alterations observed in the present study which concurred with the report of (Birkenheuer et al., 1999, Ayoob et al., 2010, Irwin et al., 2005). The destruction of circulating RBC by auto antibodies which are directed against infected and non-infected red cell membranes resulting in intravascular and extravascular haemolysis. According to Taboada and Lobetti, (2006), direct parasitic damage contributes to anaemia. A low RBC count, $\mathrm{PCV}$ and $\mathrm{Hb}$ concentration define anemia. Severe microcytic-hypochromic anemia may have been initiated by antibody mediated cytotoxic destruction of erythrocytes and/or by auto-antibody directed against components of the membranes of infected and uninfected erythrocytes which has also been reported previously in B. gibsoni infection (Aysul et al., 2013).

In the present study elevation in mean alanine aminotransferase (ALT) was noticed, which was correlated with Aysul et al., (2013) findings. Aysul et al., (2013) reported that in dogs suffering from babesiosis, elevation of bilirubin, ALT and alkaline phosphatase levels could be seen with hepatic hypoxia.

Acute renal failure in canine babesiosis (Schoeman, 2009) might have resulted into increase BUN and creatinine both $B$. gibsoni and $B$. canis infected dogs. According to Amie (2009) increased non-insulin mediated glucose consumption believed to be induced by inflammatory mediators, more especially in macrophage-rich tissues like the spleen, liver and the lungs was the cause of hypoglycaemia in the affected dogs and at the same time regarded it as a poor prognostic indicator.

Elevation of bilirubin, ALT and AKP are indicative of hepatic hypoxia and increase BUN and creatinine are indicative of degenerative changes in kidneys. Results of this study suggest that haemato-biochemical changes could be beneficial in determination of the severity of babesiosis in dogs. 
The use of molecular characterization to identify Babesia species highlights the value of procedure of PCR as an adjuvant to current diagnostic methodology.

\section{References}

Amie, K., 2009. Hypoglycemia. Small anim. Crit. Care Med. 69:295-299.

Ayoob, A.L., Hackner, S.G. and Prittie, J. 2010. Clinical management of canine babesiosis. J. Vet. Emerg. Critical Care. 20(1): 77-89.

Aysul, N., Ural, K., Ulutas, B., Eren, H. and Karagenc, T. 2013. First detection and molecular identification of Babesia gibsoni in two dogs from the Aydin province of Turkey. Turkish J. Vet. Anim. Sc, 37(2): 226-229.

Birkenheuer, A.J., Levy, M.G., Savary, K.C.M., Gager, R.B. and Breitschwerdt, E.B. 1999. Babesia gibsoni infections in dogs from North Carolina. J. Am. Anim. Hosp. Assoc. 35(2): 125-128.

Conrad, P., Thomford, J., Yamane, I., Whiting, J., Bosma, L., Uno, T., Holshuh, H.J. and Shelly, S.1991. Hemolytic anemia caused by Babesia gibsoni infection in dogs. $J$. Am. Vet Med. Assoc.199 (5):601-5.

Filipe, D.T. and Luciana, A.F. 2006. Canine babesiosis: A Brazilian persepective. Vet.Parasit. 141(3-4): 197-203.

Irwin, P.J., 2005. Babesiosis and Cytauxzoonsis. Arthropode-Borne Infectious Diseases of Dogs and Cats, $1^{\text {st }}$ edition. Manson Publishing Ltd., Barcelona, Spain,

Jacobson, L.S. and Clark, I.A. 1994. The pathophysiology of canine babesiosis: new approaches to an old puzzle. J. South Afr.
Vet. Assoc. 65(3): 134-145.

Kledmanee, K., Suwanpakdee, S., Krajangwong, S., Chatsiriwech, J., Suksai, P., Suwannachat, P., Sariya, L., Buddhirongawatr, R., Charoonrut, P. and Chaichoun, K. 2009. Development of multiplex polymerase chain reaction for detection of Ehrlichia canis, Babesia spp and Hepatozoon canis in canine blood. South Asi. J. Trop. Med. Public Health. 40: 35-39.

Lobetti, R.G., 1998. Canine babesiosis. Compend Contin. Educ. Pract. Vet. 20:418430.

Meinkoth, J.H., A.A.Kocan, S.D. Loud. and M.D.Lorenz. 2002. Clinical and hematologic effects of experimental infection of dogs with recently identified Babesia gibsoni-like isolates from Oklahoma. J. Am. Vet. Assoc. 220:185-189. Schoeman, J.P., 2009. Canine babesiosis. Onderstepoort. J. Vet. Res. 76:59-66.

Taboada, J. and S.R.Merchant. 1991. Babesiosis of companion animals and man. Vet. Clin. North Am. Small Anim. Pract. 21:103-123.

Taboada, J., 1998. Babesiosis. In: Greene CE (Ed): Infectious diseases of the dog and cat. Philadelphia, WB Saunders. Pp. 473-481.

Taboada, J. and Lobetti, R. 2006. Babesiosis. In: Infectious Diseases of the Dog and Cat. Greene, C.G. (Ed.), Elsevier, 3rd edition.

Wozniak, E.J., Barr, B.C., Thomford, J.W., Yaamanel, I., McDonough, S.P. Moore, P.F., Naydan, D., Robinson. T.W. and Conrad, P.A. 1997. Clinical, anatomic, and immunopathologic characterization of Babesia gibsoni infection in the domestic dog (canis familiaris). J. parasitol. 83(4): 692-699.

\section{How to cite this article:}

Juripriya Brahma, D. Chandrasekaran, M.G. Jayathangaraj, S. Vairamuthu and Soundararajan, C. 2019. Clinical, Haemato-Biochemical and Molecular Findings of Babesiosis in Dogs. Int.J.Curr.Microbiol.App.Sci. 8(01): 2127-2132. doi: https://doi.org/10.20546/ijcmas.2019.801.222 